\title{
Microestructura y propiedades mecánicas de materiales compuestos de matriz Al-Mg-Si-Cu reforzada con AINp, procesados por extrusión de polvos ${ }^{(\bullet)}$
}

\author{
José Luis Ortiz", Vicente Amigó ${ }^{* *}, M^{a}$ Dolores Salvador** y Claudia Roxana Pérez $z^{* * *}$
}

Resumen Se presenta una investigación experimental sobre la relación entre la estructura y las propiedades mecánicas de materiales obtenidos mediante la extrusión en caliente de preformas de polvos compactados en frío y cuya matriz es una aleación $\mathrm{Al}-\mathrm{Mg}$-Si-Cu con composición cercana a la AA6061, reforzada con tres proporciones diferentes (5, 10 y 15 $\%$ en volumen) de partículas de AIN. Se utilizó Calorimetría Diferencial de Barrido y Dilatometría sobre las muestras solubilizadas y templadas para complementar el estudio del efecto de las partículas sobre el envejecimiento de la aleación base. Se establece que es posible producir, a bajo costo, materiales compuestos de matriz metálica, MMCs, de matriz de aleación AA6061 reforzada con partículas de AlN con baja porosidad y buenas propiedades mecánicas, mediante la extrusión de mezclas de polvos compactados en frío, sin necesidad de enlatado. Sin embargo, las aglomeraciones de partículas, para porcentajes de refuerzo del orden del $15 \%$, provocan disminución de tales propiedades. Además, se plantea la posibilidad de aplicación de las técnicas dilatométricas en el estudio de los procesos de envejecimiento de las aleaciones de aluminio, con o sin la adición de refuerzos.

Palabras clave Extrusión. Consolidación de polvos. Aleaciones de aluminio. Envejecimiento. Materiales compuestos.

\section{Microstructure and mechanical properties of Al-Mg-Si-Cu matrix composites reinforced with AINp, processed by extrusion of powders}

Abstract

Keywords
This article presents an experimental investigation on the structure and mechanical properties of an $\mathrm{Al}-\mathrm{Mg}-\mathrm{Si}-\mathrm{Cu} \mathrm{P} / \mathrm{M}$ alloy reinforced with $5 \%, 10 \%$ and $15 \%$ aluminum nitride, produced by extrusion of cold compacted powders mixtures. Mechanical properties in "as extruded" and T6 conditions are compared. Differential Scanning Calorimetry and Dilatometric analysis were conducted to gain further insight into the precipitation process of these materials. Low cost $6061 \mathrm{Al} / \mathrm{AlNp}$ composites can be produced with rare and small porosity by extrusion of cold compacted shapes without canning. The mechanical properties of the MMCs obtained by this process have limitations for high particles fractions because of clustering effects. All materials are always harder than the matrix and shows a similar behavior during aging processes but kinetics is changed. Potential applications of dilatometric techniques in the aging investigations of aluminum alloys and aluminum matrix composites have been established.

Extrusion. Powder consolidation. Aluminum alloys. Aging. Metal matrix composites.

(•) Trabajo recibido el día 18 de julio de 2000 y aceptado en su forma final el día 7 de noviembre de 2000.

(*) Departamento de Ingeniería Mecánica e Industrial. Instituto Tecnológico y de Estudios Superiores de Monterrey (ITESM). Campus Querétaro. Epigmenio González, 500.76130 Santiago de Querétaro (Méjico).

(**) Departamento de Ingeniería Mecánica y de Materiales. Universidad Politécnica de Valencia (UPV). Camino de Vera, s/n. 46022 Valencia (España).

${ }^{* * *}$ Instituto de Tecnología "Jorge A. Sabato". Universidad Nacional de General San Martín (UNISAM)-Comisión Nacional de Energía Atómica (CNEA). Avda. General Paz, 1499. San Martín, Buenos Aires (Argentina). 


\section{INTRODUCCIÓN}

Las aleaciones Al-Mg-Si (6XXX) se emplean extensamente en productos de forja debido a que presentan una combinación de propiedades que las hacen adecuadas para un gran número de aplicaciones, al poseer una resistencia moderadamente alta después de aplicarles un tratamiento térmico (generalmente T6), una buena resistencia a la corrosión y excelente conformabilidad. Lo anterior, las hace más adecuadas que las aleaciones de las familias 2 XXX y $7 X X X$, de mayor resistencia para muchas aplicaciones ${ }^{[1]}$. En las aleaciones Al-Mg-Si, el magnesio y el silicio se combinan para formar el siliciuro de magnesio $\left(\mathrm{Mg}_{2} \mathrm{Si}\right)$ cuya microestructura puede discutirse a través de su diagrama pseudobinario con el aluminio. El porcentaje de siliciuro de magnesio en las aleaciones comerciales oscila entre 0,6 y $1,5 \%$ en peso ${ }^{[2]}$. Se ha encontrado ${ }^{[3]}$ que las aleaciones pobres, esto es, aquellas cuyas composiciones se encuentran en los límites inferiores de composición química dentro de las especificaciones estándar, desarrollan una mejor combinación entre resistencia, ductilidad y tenacidad a la fractura cuando se emplean en la fabricación de materiales compuestos.

Las consideraciones costo/propiedades han concentrado la atención en aplicaciones comerciales de los materiales compuestos de matriz metálica con refuerzos discontinuos, como las aleaciones de aluminio reforzadas ya sea con whiskers de carburo de silicio, partículas de carburo de silicio o de alúmina, sílice o de diversos nitruros ${ }^{[4-16]}$.

El primer paso en la secuencia de fabricación de materiales compuestos de matriz metálica (MMCs) con refuerzos discontinuos (partículas, en este caso) es la selección adecuada del refuerzo y de la aleación de la matriz. En la mayoría de los casos, los principales criterios de selección involucran cuatro aspectos fundamentales: propiedades, fabricabilidad, costo y disponibilidad. Budinski ${ }^{[17]}$ y Asbhy y Waterman ${ }^{[18]}$ proporcionan una descripción detallada de las metodologías de selección de materiales constituyentes para un material compuesto. Mientras que los requerimientos de propiedades físicas y mecánicas pudieran limitar las alternativas de selección, la reactividad química de los refuerzos cerámicos, ya sea durante la fabricación o durante el servicio, frecuentemente determinará la combinación final refuerzo-matriz. Algunos trabajos de investigación ${ }^{[15]}$ sugieren, sin embargo, que es posible desarrollar zonas de reacción matriz/refuerzo. Por ejemplo, el $\mathrm{SiC}$ posee excelente resistencia mecánica y módulo elástico, pero es termodinámicamente inestable en las aleaciones de aluminio a elevada temperatura. La aplicación de recubrimientos adecuados a los materiales de refuerzo ${ }^{[15]}$, ofrece una posible alternativa para combinar estos refuerzos cerámicos con matrices de aluminio, especialmente cuando éstas deban exponerse a altas temperaturas. Sin embargo, investigaciones relativamente recientes ${ }^{[19]}$ demuestran que al emplear AlN sin tratamiento superficial como material de refuerzo, se logran resistencias a la tracción similares a las de los materiales reforzados con fracciones volumétricas semejantes de $\mathrm{SiC}$, con la salvedad de que el comportamiento de los primeros materiales es dúctil a elevadas temperaturas $\left(\approx 400^{\circ} \mathrm{C}\right)$ mientras que el segundo grupo de materiales exhibe fractura frágil debido a la descohesión entre las partículas $\mathrm{SiC}$ y la matriz. Además, se ha observado una mayor resistencia a elevadas temperaturas de los materiales Al/AlN como consecuencia de la bajísima reactividad entre refuerzo y matriz, en contraste con el incremento de reactividad con la temperatura, entre el $\mathrm{SiC}$ y la matriz de aluminio, especialmente cuando se obtiene por colada el material compuesto. Este mismo estudio demostró que los materiales $\mathrm{Al} / \mathrm{Al}_{2} \mathrm{O}_{3}$ tenían menor resistencia y ductilidad que los materiales reforzados con las mismas fracciones volumétricas de $\mathrm{SiC}$ y $\mathrm{AlN}$, por lo que se concluye que el empleo de AlN como material de refuerzo constituye una forma efectiva de evitar las reacciones indeseables en las intercaras.

Daniel y Murthy ${ }^{[20]}$ han determinado que las propiedades mecánicas de los MMCs reforzados con partículas, obtenidos por oxidación o nitruración in situ de la aleación fundida $\left(\mathrm{Al}_{2} \mathrm{O}_{3} / \mathrm{Al}\right.$ y AlN/Al, respectivamente) son comparables entre sí y con los materiales obtenidos por la adición física del refuerzo. Sin embargo, el segundo grupo de materiales exhibe una mayor conductividad térmica y sensibilidad a la humedad. Asimismo, indican que aunque las propiedades mecánicas del $\mathrm{Al}_{2} \mathrm{O}_{3}$ y el AlN son bastante similares, el AlN tiene una mayor conductividad térmica (diez veces mayor) y menor coeficiente de expansión térmica $(\alpha)$ que el $\mathrm{Al}_{2} \mathrm{O}_{3}$. Adicionalmente, el AlN tiene excelente resistencia a la corrosión y buena mojabilidad con el aluminio.

La única limitación del AIN es que se hidroliza, liberando amoniaco y calor, originando un deterioro de sus propiedades mecánicas a largo plazo ${ }^{[21]}$.

La respuesta al envejecimiento de los MMCs dependerá de toda una gama de factores, que incluyen a la matriz en particular, al refuerzo, la 
historia de procesamiento del compuesto y la temperatura y tiempo de envejecimiento. Sin embargo, debe destacarse que a pesar de cualquier modificación en la cinética de envejecimiento de los compuestos, las propiedades óptimas de envejecimiento, se obtienen generalmente con la misma práctica comercial de envejecimiento utilizada para las aleaciones sin refuerzo, particularmente en los materiales compuestos procesados en estado líquido. Al respecto, T. Das et. al. ${ }^{[4]}$ han realizado estudios sobre el efecto de partículas cerámicas de $\mathrm{Al}_{2} \mathrm{O}_{3}$ de formas esféricas y angulares, sobre el comportamiento durante la precipitación de los MMCs, en matrices de aleaciones de aluminio AA6061, usando Calorimetría Diferencial de Barrido (CDB), Microscopía Electrónica de Transmisión (MET) y mediciones de microdureza. Encontraron que, aunque se presenta una aceleración de la cinética de disolución y precipitación, no todas las etapas del proceso de envejecimiento de estas aleaciones se ven influidas por la presencia de partículas. El grado de aceleración y las proporciones relativas de fases dependen de parámetros de refuerzo, tales como tamaño, forma y fracción volumétrica. En los MMCs observaron mayores densidades de dislocaciones en las vecindades de las partículas cerámicas, causadas, probablemente, por las tensiones residuales acumuladas en estas zonas como consecuencia de las grandes diferencias en los valores de $\alpha$, entre las partículas cerámicas y la matriz. Estos efectos de las dislocaciones $\alpha$ influyen en la cinética de precipitación.

\section{PROCEDIMIENTO EXPERIMENTAL}

Los materiales compuestos para este estudio consisten en mezclas de nitruro de aluminio, en tres porcentajes en volumen $(5,10$ y $15 \%)$, con una matriz de aleación de aluminio, cuya composición química es cercana a la aleación de forja AA6061 con bajo porcentaje de magnesio y mayor contenido de cobre. La composición química del refuerzo es: hierro: 200 ppm; silicio: 99 ppm; otros metales: 34 ppm; carbono total: 0,01\%; tamaño de partícula: $\mathrm{d}_{50}=8 \mu \mathrm{m}, \mathrm{d}_{97}=19 \mu \mathrm{m}$; y fue suministrada por Advanced Refractory Technologies (ART) de Buffalo, Nueva York. La aleación de aluminio fue suministrada a su vez por The Aluminium Powder Co. Ltd. de Midland, Inglaterra. La composición química en porcentajes en peso es: $\mathrm{Fe},<0,2 ; \mathrm{Si}$, 0,65; Cu, 0,87; Cr, 0,23. Su tamaño máximo de partícula es de $75 \mu \mathrm{m}$. La figura 1 muestra fotomicrografías de Microscopía Electrónica de Barrido
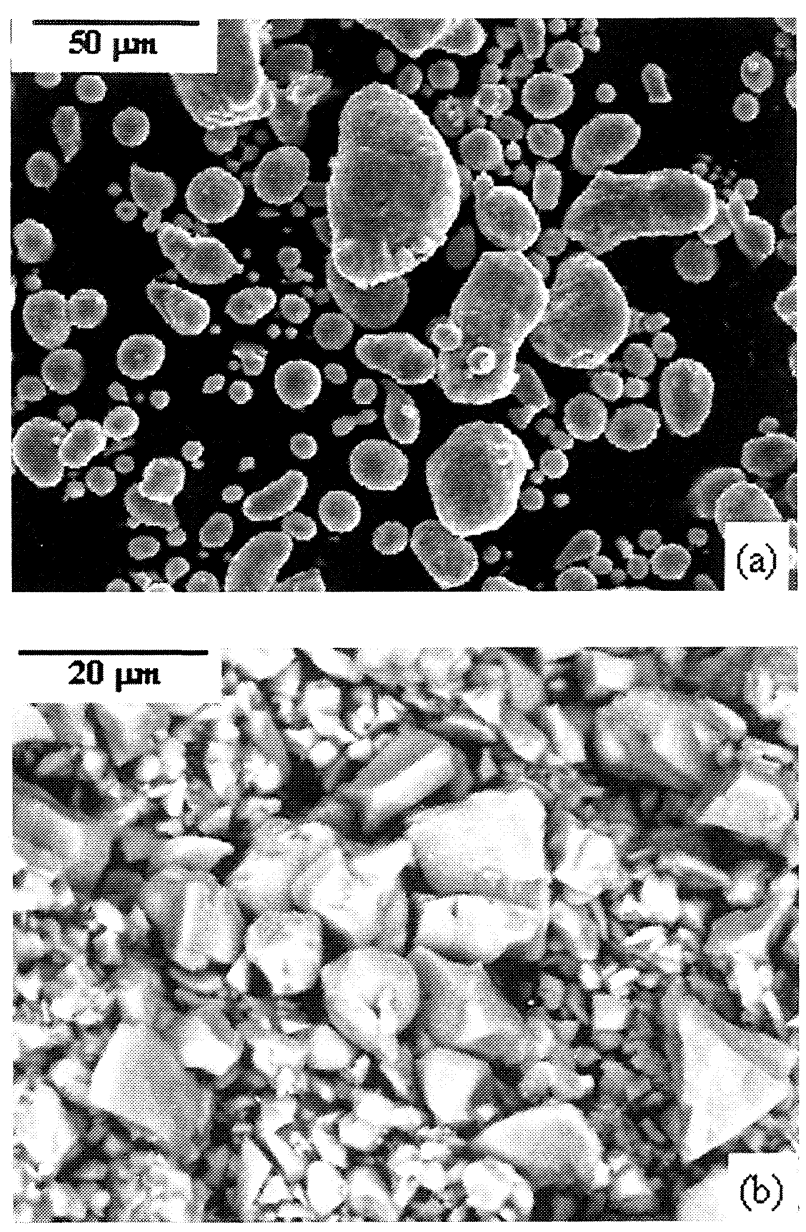

Figura 1. Micrografías MEB de los polvos. a) Aleación de Aluminio "6061". b) De nitruro de aluminio.

Figure 1. SEM Micrographs of the. a) "6061" and Al Alloy. b) Aluminum Nitride powders.

(MEB) del polvo atomizado de la aleación base, con forma redondeada (a) y de los polvos de nitruro de aluminio, con forma poligonal (b). Después del mezclado y homogeneización de los polvos en un molino de laboratorio, de bolas de alúmina, durante dos horas a $3 \pi \mathrm{rad} / \mathrm{s}$, las mezclas se sometieron a compactación uniaxial en frío con aplicación lenta y gradual de la presión hasta $250 \mathrm{MPa}$, obteniéndose compactos cilíndricos de $2,5 \mathrm{~cm}$ de diámetro por $3 \mathrm{~cm}$ de altura, aproximadamente. Estos se extruyeron a $550^{\circ} \mathrm{C}$, con una velocidad de desplazamiento del pistón de $1 \mathrm{~mm} / \mathrm{s}$ a través de una matriz cónica cuya sección circular tiene un diámetro final de $5 \mathrm{~mm}$ y ángulo de ataque de $120^{\circ}$. Finalmente, se enfriaron en aire quieto a temperatura ambiente, obteniéndose de esta forma, varillas de, aproximadamente, $5 \mathrm{~mm}$ de diámetro y 400 $\mathrm{mm}$ de longitud con un acabado superficial terso. Como material de referencia, se consolidó la aleación base sin reforzar por el mismo procedimiento. 
Las muestras para el tratamiento T6 se cortaron de las mismas varillas y se solubilizaron a $530{ }^{\circ} \mathrm{C}$ durante $1,5 \mathrm{~h}$, enfriándolas a temperatura ambiente por inmersión en agua y envejecimiento inmediato a $175^{\circ} \mathrm{C}$ durante $8 \mathrm{~h}$.

Se obtuvieron y analizaron muestras para microscopía óptica $(\mathrm{MO})$, microscopía electrónica de barrido (MEB) y de transmisión (MET), durezas Vickers (carga: $10 \mathrm{~N}$, durante $15 \mathrm{~s}$ ) y ensayos de tracción. Se realizaron tratamientos de envejecimiento a $150^{\circ} \mathrm{C}$ durante diferentes tiempos, determinando las durezas, y se prepararon muestras para metalografía convencional. Las preparaciones de las muestras para microscopía electrónica de transmisión (MET) se han realizado mediante pulido electrolítico con un equipo Tenupol de Struers, utilizando como electrolito una solución al $20 \%$ de ácido nítrico a $-20^{\circ} \mathrm{C}$. Las muestras de han observado en un microscopio de transmisión-barrido de Hitachi de 200 kV, realizándose el análisis de los
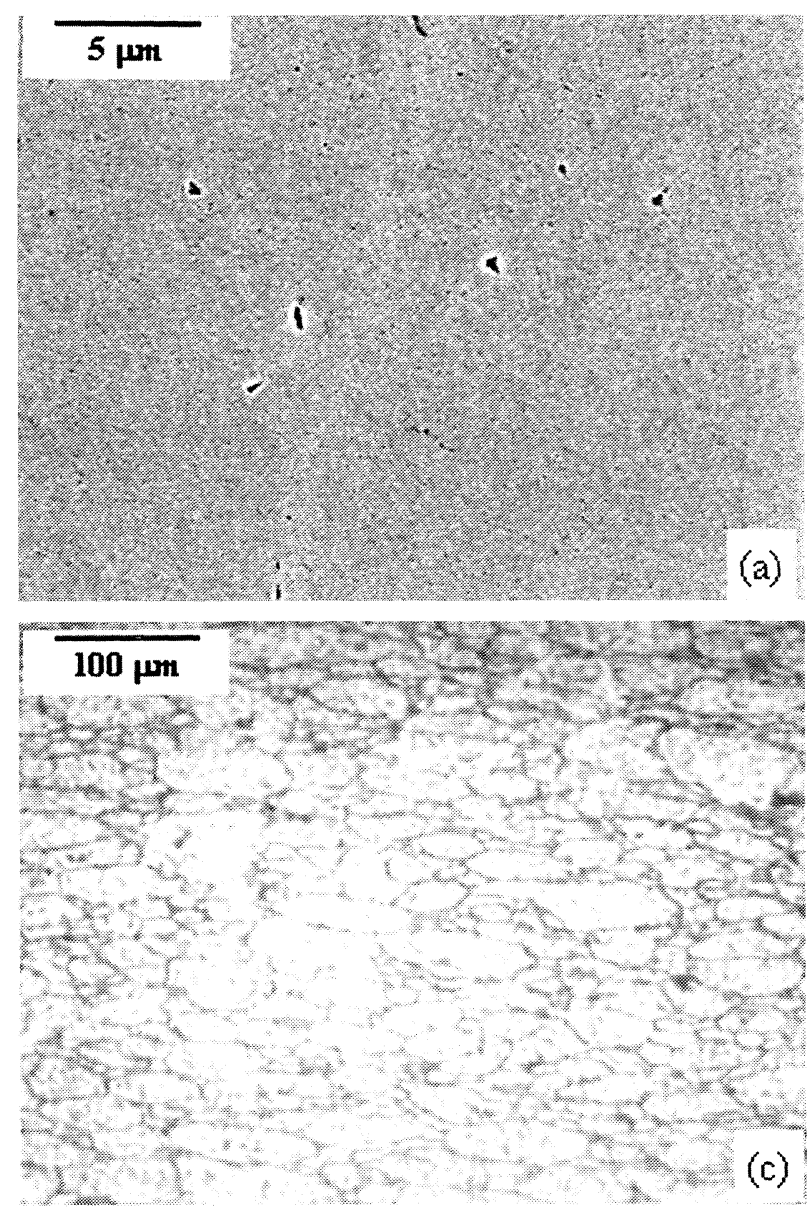

precipitados mediante el análisis de energías dispersivas de rayos $\mathrm{X}$.

A fin de analizar con mayor detalle el proceso de envejecimiento, se determinaron las curvas de calorimetría diferencial de barrido (CDB) de las muestras solubilizadas y templadas. Los barridos se realizaron en una atmósfera protectora de argón a $20^{\circ} \mathrm{C} / \mathrm{min}$, siendo las muestras pequeños botones de aproximadamente $50 \mathrm{mg}$, usando aluminio de $99,998 \%$ de pureza como material de referencia.

Finalmente, se determinaron las variaciones longitudinales de muestras de $25 \mathrm{~mm}$ solubilizadas y templadas mediante Dilatometría Diferencial a la misma velocidad de barrido.

\section{RESULTADOS}

La figura 2 muestra fotomicrografías de la aleación 6061 después de la extrusión, donde pueden
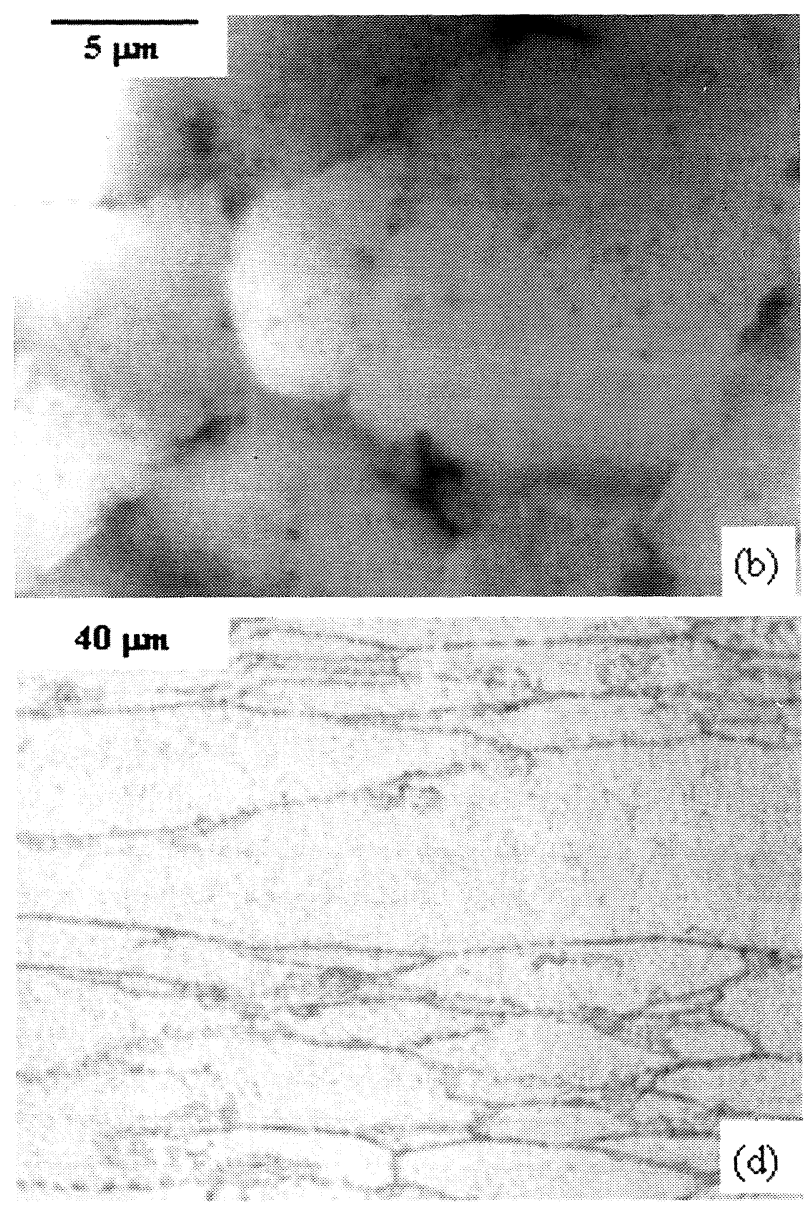

Figura 2. Micrografías de la aleación "6061". a) Extruída, (corte transversal MEB). b) Extruída, (corte transversal MET) (los óxidos se marcan con las flechas). c) Compactada, (justo antes de la reducción de área durante la extrusión MO). d) Extruída y sobreenvejecida, (corte longitudinal MO).

Figure 2. Micrographs of the "6061 Al" Alloy : a) extruded, (transversal section SEM). b) extruded, (transversal section TEM), (arrows mark oxides). c) compacted, (just before the section reduction during extrusion OM). d) extruded and overaged, (longitudinal section $\mathrm{OM}$ ). 
observarse una escasa y fina porosidad (a) y una capa de óxidos e hidróxidos que se ha fracturado después de la compactación y extrusión y, cuyos fragmentos permanecen en las superficies originales de los polvos después del proceso (Fig. 2 b). En la figura $2 \mathrm{c}$ ), se observa la fotomicrografía de un corte longitudinal de la matriz, cuando ésta es compactada en caliente, justamente antes de pasar por el dado de extrusión y, en la Fig. 2 d) se muestra la fotomicrografía de un corte longitudinal de la matriz extruída, después de un sobreenvejecimiento a $150{ }^{\circ} \mathrm{C}$ durante $116 \mathrm{~h}$, donde puede observarse la orientación de los granos en la dirección de extrusión y una distribución preferencial de las partículas de óxido y precipitados en los límites de grano. La figura 3 muestra fotomicrografías ópticas de las secciones transversales de las varillas de los MMCs con $5 \%$ (a), $10 \%$ (b) y $15 \%$ (c) de refuerzo después de la extrusión, en las que se aprecia una distribución razonablemente uniforme de las partículas de AlN. No obstante, puede percibirse un grado de aglomeración creciente a medida que se incrementa el contenido de AIN. La figura 4 muestra una fotomicrografía de la sección longitudinal de una muestra con $10 \%$ de AlN, después de haberse sometido a un proceso de sobreenvejecimiento a $150{ }^{\circ} \mathrm{C}$ durante $116 \mathrm{~h}$, posterior a una solubilización a $530^{\circ} \mathrm{C}$ durante $1,5 \mathrm{~h}$ y temple en agua a $25^{\circ} \mathrm{C}$. Se observa el flujo plástico del material alrededor de las partículas de refuerzo. La tabla I muestra los valores de resistencia a la tracción, límite elástico al 0,2\% y alargamiento de rotura para los materiales procesados, tanto después de la extrusión con enfriamiento al aire como después de haberse sometido al tratamiento de envejecimiento T6. La figura 5 muestra los valores de dureza Vickers de los materiales compuestos de matriz metálica y de la matriz, después de envejecer las muestras a $150^{\circ} \mathrm{C}$ durante varios tiempos. La figura 6 muestra los termogramas CDB de la matriz y de los MMCs solubilizados y templados. Puede apreciarse que todos los materiales muestran un comportamiento similar durante la precipitación, con la manifestación de dos picos exotérmicos a $\sim 250^{\circ} \mathrm{C}$ (A) y a $\sim 300^{\circ} \mathrm{C}(\mathrm{B})$. La tabla II muestra los valores obtenidos para las temperaturas y entalpías de los picos calculados a partir de la gráfica anterior. La figura 7 muestra las curvas dilatométricas de la matriz y de los MMCs después del proceso de solubilización y temple y la figura 8 presenta las curvas de variación de a con la temperatura para estos materiales, representado en intervalos de $5{ }^{\circ} \mathrm{C}$, en los que puede apreciarse una disminución de los
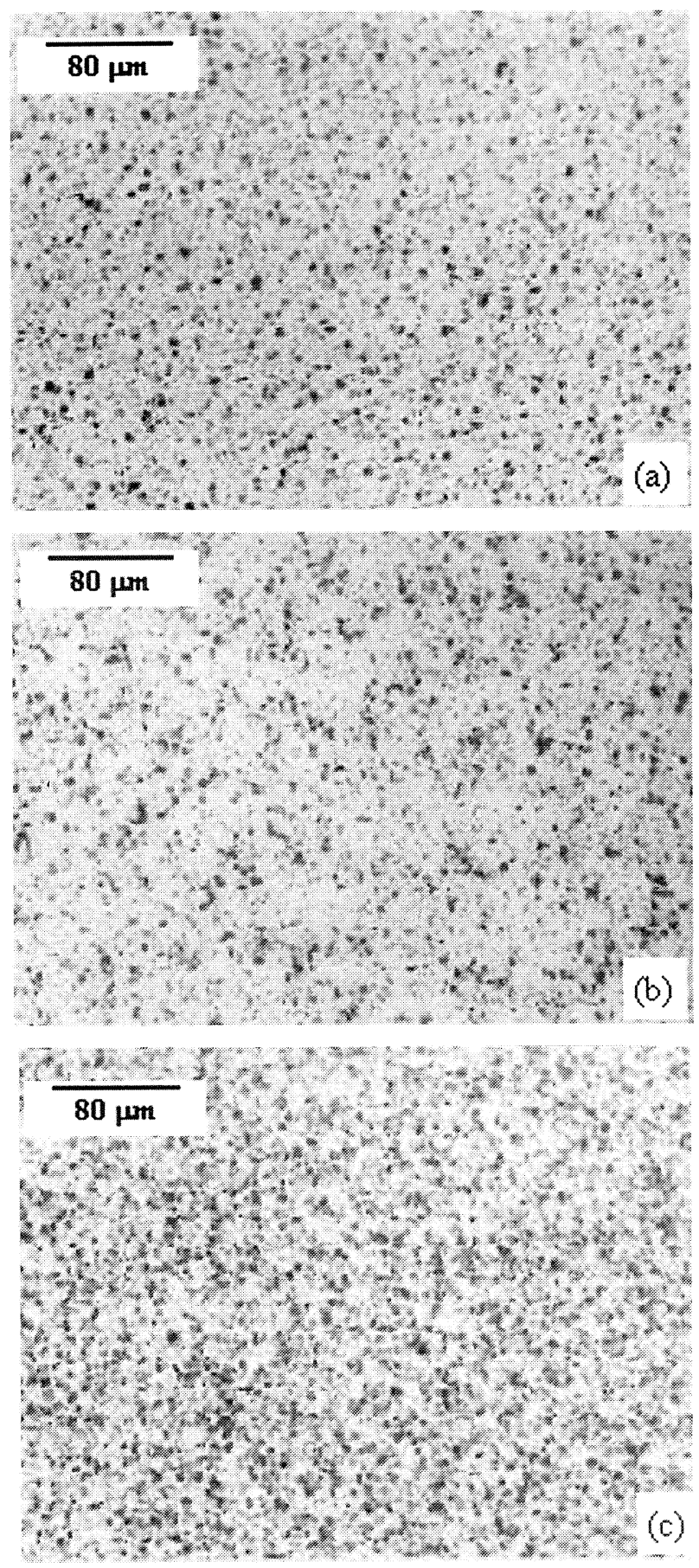

Figura 3. Micrografías ópticas de las secciones transversales de los MMCs extruídos con: a) $5 \%$ AIN. b) $10 \%$ AIN. c) 15 $\%$ AIN.

Figure 3. OM micrographs of the transversal sections of the extruded MMCs with: a) $5 \%$ AIN. b) $10 \%$ AIN. c) $15 \%$ AIN.

valores de $\alpha$ en el intervalo de $\sim 230^{\circ} \mathrm{C}$ a $\sim 320^{\circ} \mathrm{C}$, que es más intensa para la aleación sin reforzar.

\section{DISCUSIÓN}

Los resultados obtenidos proporcionan información sobre la influencia de la fracción en volumen 


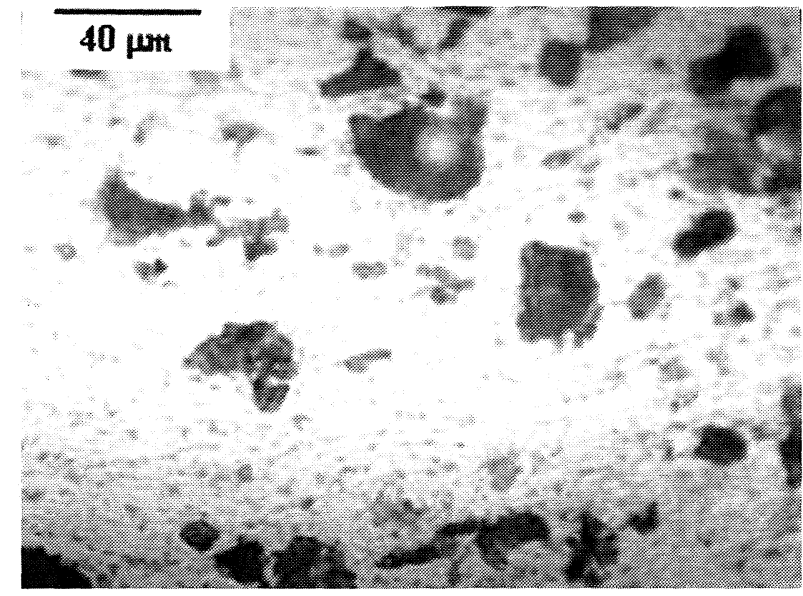

Figura 4. Micrografía óptica del MMC con $10 \%$ de AIN sobreenvejecido.

Figure 4. OM micrograph of the overaged MMC with $10 \%$ AIN.

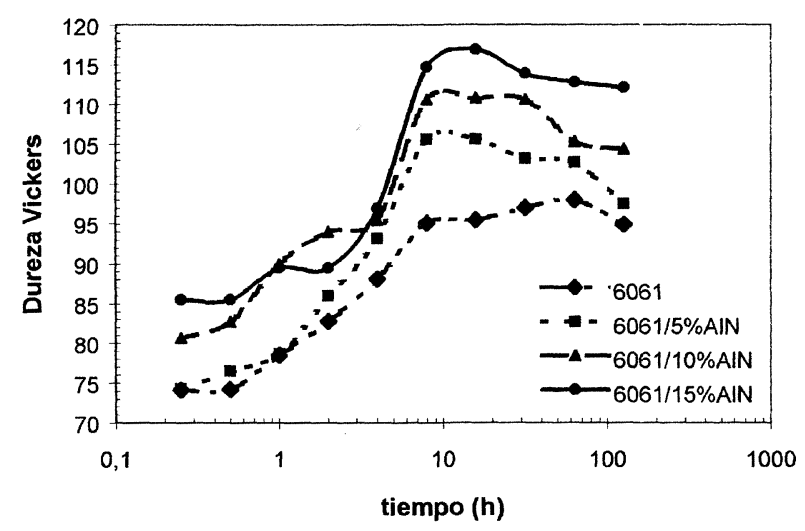

Figura 5. Variación de la dureza Vickers con el tiempo de Envejecimiento a $150^{\circ} \mathrm{C}$.

Figure 5. Hardness Vickers variation at aging time of $150^{\circ} \mathrm{C}$

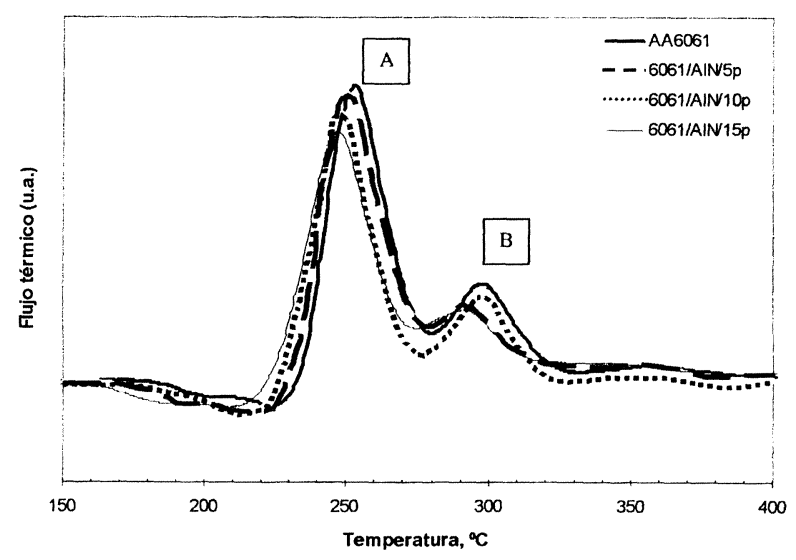

Figura 6. Termogramas CDB de la matriz y de los MMCs solubilizados y templados.

Figure 6. DSC Thermograms DSC for the alloy matrix and the MMCs solutionized and quenched.

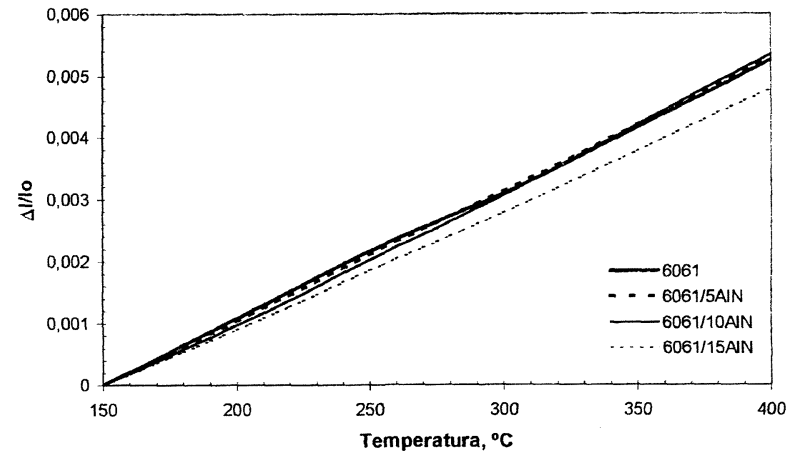

Figura 7. Curvas dilatométricas de la matriz y de los MMCs obtenidas después de solubilización y temple.

Figure 7. Dilatometric graphs of the matrix and MMCs obtained after solutionized and quenching.

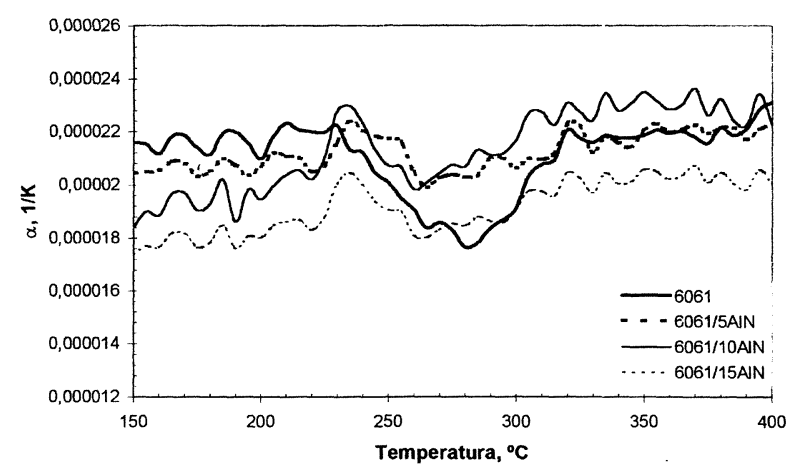

Figura 8. Curvas de variación de a con la temperatura para muestras solubilizados y templados.

Figure 8. a vs. Temperature variations for the solutionized and quenched specimens.

de AIN en las propiedades mecánicas para bajos contenidos de refuerzo de materiales 6061/AlNp. Estas propiedades dependen, en gran medida, de la distribución de partículas y, en menor grado, del porcentaje de refuerzo (Tabla I y Fig. 3). Se puede observar que, para contenidos de 10 y $15 \%$ en volumen, las propiedades de tracción se reducen para las muestras extruidas. Este hecho probablemente refleje problemas de mezclado, al no obtenerse una distribución uniforme de las partículas de matriz y refuerzo para mayores contenidos de este último, lo cual produce un mayor número de sitios (y de mayor tamaño) para nucleación de grietas como resultado de la aglomeración de partículas de AIN (Fig. 3).

La aleación de aluminio empleada contiene un reducido porcentaje de $\mathrm{Mg}$ (sólo del 0,17\%) con una relación $\mathrm{Mg} / \mathrm{Si}$ muy baja, por lo que podría esperarse valores de resistencia y dureza inferiores a los promedios para esta aleación de forja. Sin embargo, esto no sucede y los valores de 
Microestructura y propiedades mecánicas de materiales compuestos...

Tabla I. Propiedades mecánicas de los materiales extruidos y con el tratamiento T6

Table I. Mechanical properties of the extruded materials and with T6 treatment

\begin{tabular}{lcccccc}
\hline \multirow{2}{*}{ Material } & \multicolumn{3}{c}{ Extruido } & & T6 \\
\cline { 2 - 7 } & $\begin{array}{c}\text { Resistencia a } \\
\text { la tracción } \\
(\mathrm{MPa})\end{array}$ & $\begin{array}{c}\text { Límite elástico } \\
0,2 \%\end{array}$ & $\begin{array}{c}\text { Alargamiento } \\
(\mathrm{MPa})\end{array}$ & $\begin{array}{c}\text { Resistencia a } \\
\text { la tracción } \\
(\mathrm{MPa})\end{array}$ & $\begin{array}{c}\text { Límite elástico } \\
0,2 \% \\
(\mathrm{MPa})\end{array}$ & $\begin{array}{c}\text { Alargamiento } \\
\%\end{array}$ \\
\hline 6061 & 193 & 103 & 31,2 & 306 & 181 & 11,1 \\
$6061 / 5 \%$ AIN & 209 & 112 & 24,0 & 292 & 187 & 8,5 \\
$6061 / 10 \%$ AIN & 207 & 93 & 15,4 & 283 & 192 & 7,6 \\
$6061 / 15 \%$ AIN & 182 & 93 & 11,3 & 265 & 191 & 4,0 \\
\hline
\end{tabular}

Tabla II. Temperaturas y energías de los picos de los termogramas de la Fig. 6

Table II. Temperatures and energies of the peaks in the Fig. 6 thermograms

\begin{tabular}{|c|c|c|c|c|}
\hline \multirow[t]{2}{*}{ Material } & \multicolumn{2}{|c|}{ Pico A } & \multicolumn{2}{|c|}{ Pico B } \\
\hline & $\mathrm{T},{ }^{\circ} \mathrm{C}$ & $\Delta \mathrm{H}, \mathrm{J} / \mathrm{g}$ & $\mathrm{T},{ }^{\circ} \mathrm{C}$ & $\Delta \mathrm{H}, \mathrm{J} / \mathrm{g}$ \\
\hline 6061 & 252,1 & 7,43 & 297,8 & 1,50 \\
\hline $6061 / 5 \%$ AIN & 258,1 & 7,46 & 308,7 & 1,63 \\
\hline $6061 / 10 \%$ AIN & 254,7 & 7,27 & 298,7 & 0,72 \\
\hline $6061 / 15 \%$ AIN & 261,6 & 6,69 & 306,8 & 0,71 \\
\hline
\end{tabular}

propiedades mecánicas de la matriz se encuentran dentro de los rangos especificados para el material de forja (Tabla I). Así que, es posible establecer que el proceso de extrusión en caliente, sin enlatado de preformas de polvos a los que se les ha formado una delgada capa de óxidos e hidróxidos es un proceso adecuado, cuando las relaciones de extrusión son lo suficientemente elevadas como para producir ruptura de esta capa, debido a las elevadas deformaciones de corte generadas y una buena coalescencia de los polvos metálicos con una porosidad escasa y de tamaño pequeño, lo cual conduce a densidades superiores al 99 \% de la teórica (Fig. 2a). La distribución más homogénea de las partículas de óxido en los MMCs que en la matriz puede ser atribuida a su mejor dispersión e incorporación en el material, como consecuencia del flujo del metal alrededor de las partículas de AIN durante la extrusión, que arrastra a los restos de óxido, describiendo trayectorias que tienden a rodear a las partículas del refuerzo, como puede observarse en la figura 4.

Los MMCs presentan siempre valores de dureza superiores a los de la matriz, en cualquier combinación temperatura/tiempo de envejecimiento, además de un incremento en la cinética del proceso, como puede observarse en la figura 5; ya que el tiempo necesario para alcanzar el valor máximo de dureza a una temperatura de $150^{\circ} \mathrm{C}$ es significativamente menor que el necesario para alcanzarlo en la aleación sin refuerzo. Tal efecto se atribuye a la combinación de la aceleración de la cinética de precipitación ejercido por el refuerzo (por formación de dislocaciones generadas como consecuencia de las diferencias de coeficientes de expansión térmica entre la matriz metálica y el refuerzo cerámico), por efectos interfaciales y, por los precipitados generados durante el proceso de envejecimiento, ya que las diferencias de dureza se mantienen con cierta aproximación, independientemente del tiempo de envejecimiento.

La siguiente secuencia del proceso de envejecimiento de las aleaciones AA6061 es una de las más aceptadas ${ }^{[22]}$ : Solución Sólida $\alpha \rightarrow$ Zonas GuinierPreston esféricas $\rightarrow$ Precipitados metaestables coherentes $\beta$ " de forma acicular $\rightarrow$ Precipitados metaestables semicoherentes $\beta$ ' en forma de bastones $\rightarrow$ Precipitados incoherentes estables $\beta$ $\left(\mathrm{Mg}_{2} \mathrm{Si}\right)$ en forma de placas. De acuerdo con lo anterior, en la figura 9 se muestra una fotomicrografía MET donde pueden apreciarse los precipitados $\beta$ ', además de precipitados de tipo $(\mathrm{Fe}, \mathrm{Mn}$, $\mathrm{Cr})_{3} \mathrm{SiAl}_{12}$, identificados por sus energías dispersivas, después del tratamiento T6. En la figura 6 puede asociarse el pico A a la precipitación de $\beta$ " y el pico $B$ a la precipitación de $\beta$ ', observándose que la adición de las partículas de refuerzo no altera la secuencia de envejecimiento, y, sin embargo, la cinética del proceso cambia ligeramente, al producirse las transformaciones a menores temperaturas.

En la figura 7 no se observan cambios significativos en la expansión lineal de los materiales analizados. No obstante, se aprecia una disminución de los alargamientos, que se hace más acentuada a medida que se incrementa el contenido de refuerzo 


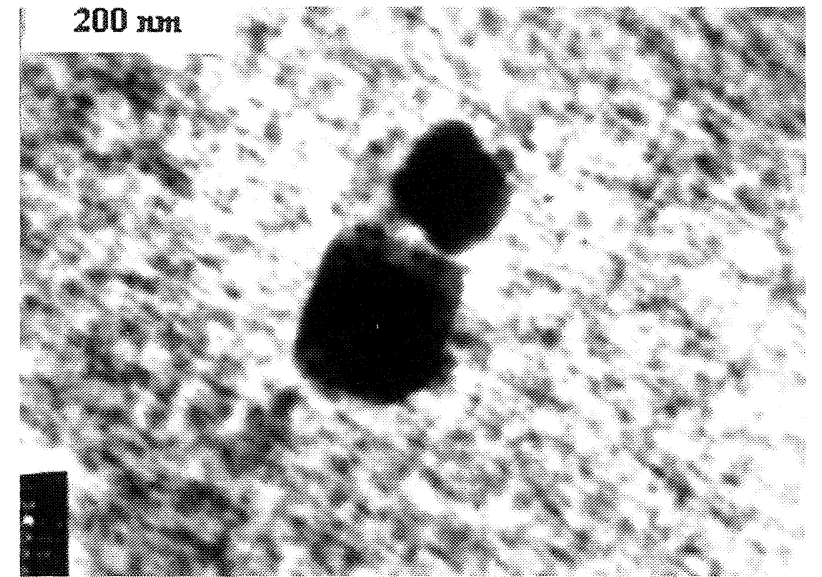

Figura 9. Micrografía MET de los precipitados presentes en la aleación base con tratamiento T6.

Figure 9. TEM micrography of the precipitaters in the matrix alloy with $T 6$ treatment.

como resultado, fundamentalmente, del menor valor de a del AlN con respecto a la aleación base. Sin embargo, no se sigue la regla de las mezclas debido a las tensiones (deformaciones) introducidas por estos cambios dimensionales heterogéneos, matriz/refuerzo. En la figura 8, en cambio, se aprecia una mayor diferencia entre los valores de $\alpha$, como consecuencia de las adiciones del refuerzo, además de una clara disminución (sobre todo en la matriz sin refuerzo), entre $\sim 230^{\circ} \mathrm{C}$ y $\sim 320^{\circ} \mathrm{C}$ para este coeficiente. Aunque las técnicas dilatométricas han demostrado ser herramientas valiosas, sobre todo para el estudio de transformaciones masivas (v. g.: transformación martensítica), en el caso del estudio del proceso de envejecimiento de aleaciones de aluminio (donde las transformaciones de fase en el estado sólido involucran solamente una fracción muy pequeña del volumen del material), podrían tener cierta aplicación. En la figura 10 se han superpuesto las Gráficas CDB y $\alpha-T$ para la aleación después de solubilización y temple, pudiendo observarse una clara correspondencia entre ambas. Los picos exotérmicos de formación de las fases $\beta$ " y $\beta$ ' coinciden con una disminución clara de $\alpha$. A medida que se van formando los precipitados coherentes, aumentan las tensiones en el material, disminuyendo su tendencia a dilatarse y, una vez formados los precipitados incoherentes, $\beta$, este efecto desaparece.

Mientras que para CDB se presentan dos picos en el intervalo de temperaturas, considerado como consecuencia de las evoluciones térmicas de los precipitados $\beta$ " y $\beta$ ', en el caso de la curva dilatométrica solamente se aprecia un pico de mayor

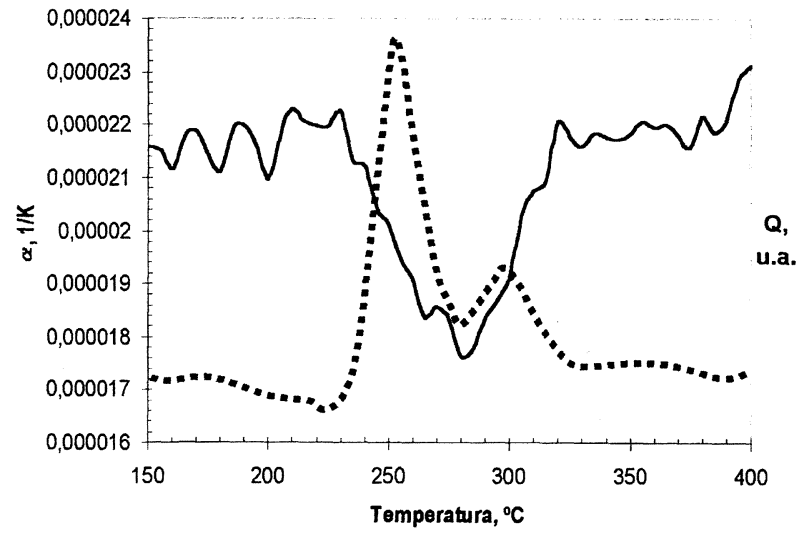

Figura 10. Gráficas CDB y a vs. T para la aleación base.

Figure 10. DSC and a vs. T graphs for the matrix alloy.

anchura que cubre el prácticamente el mismo intervalo, cuyo mínimo es coincidente con el mínimo relativo 'generado entre los dos picos exotérmicos de la curva CDB. Esto indica que el máximo nivel de esfuerzos se presenta a esta temperatura, una vez que se ha formado completamente el precipitado coherente $\beta "\left(T \sim 280^{\circ} \mathrm{C}\right)$. Al iniciarse la formación de $\beta$ ' hay una pérdida gradual de la coherencia, como consecuencia del nivel de tensiones internas acumuladas, iniciándose al mismo tiempo la recuperación del valor de $\alpha$, hasta llegar a un nivel similar al que tenía antes de iniciarse el proceso de precipitación. Este hecho es de suma importancia en la comprensión del proceso de envejecimiento de las aleaciones $\mathrm{Al}-\mathrm{Mg}-\mathrm{Si}$, ya que, todavía en la actualidad, permanece en controversia $^{[23]}$. Es apreciable una menor correlación entre los gráficos para el material compuesto (Figs. 6 y 8), lo cual se atribuye a la interferencia del refuerzo.

\section{CONCLUSIONES}

- Es posible obtener, mediante extrusión de preformas de polvos compactados uniaxialmente en frío, varillas de la aleación de composición cercana a la AA6061 y de materiales compuestos reforzados con partículas de AlN en esta matriz, con escasa y fina porosidad y densidades cercanas al $100 \%$ de la teórica.

- Las propiedades mecánicas de la aleación extruida son similares a las de los correspondientes materiales de forja, a pesar de que se tiene un contenido de $\mathrm{Mg}$ relativamente bajo y de que la película de óxidos e hidróxidos, que originalmente recubre los polvos, se haya fracturado parcialmente. 
- Las propiedades mecánicas de los MMCs obtenidos por este proceso tienen limitaciones inherentes a la cantidad de refuerzo, ya que aparece un gran número de sitios de nucleación de grietas, como resultado de la aglomeración de partículas para contenidos de refuerzo del 10 y $15 \% \mathrm{v}$ de refuerzo.

- Los MMCs tienen una distribución de óxidos y precipitados más uniforme que la matriz, como consecuencia de una mejor incorporación de sus fragmentos en el metal, debido a la ruptura del flujo plástico isotrópico por las partículas de refuerzo.

- Una distribución adecuada de partículas puede conducir a mejores propiedades mecánicas ya que es más difícil generar longitudes de grieta elevadas.

- Todos los MMCs alcanzan siempre mayores durezas y muestran un proceso de envejecimiento con una mayor cinética que la aleación base. Sin embargo, la adición de partículas no cambia la secuencia de envejecimiento de la aleación base.

- Existe correspondencia entre el comportamiento calorimétrico y dilatométrico de los materiales, que se observa con mayor claridad sin la interferencia de los efectos del refuerzo, por lo que las técnicas dilatométricas son útiles en los estudios de envejecimiento de las aleaciones de aluminio y, con menor precisión, para estos materiales compuestos.

- La correspondencia entre los picos exotérmicos $\beta$ ” y $\beta$ ' de los termogramas CDB con las gráficas $\alpha-T$ constituye una evidencia importante en la resolución de la controversia sobre la asociación de los precipitados a estos picos, durante el envejecimiento de las aleaciones Al-Mg-Si.

\section{AGRADECIMIENTOS}

Expresamos nuestro agradecimiento al CICYT de España, por su apoyo a través del proyecto MAT99. 0579-C03-01 y al CONACyT de México por la Beca al extranjero otorgada a uno de los autores.

\section{REFERENCIAS}

[1] L. ZHEN y S.B. KANG, Scripta Mater. 36 (1997) 1089-1094.
[2] J.E. Hatch, Aluminum, Properties and Physical Metallurgy, ASM, Metals Park, Oh., 1984, p. 50.

[3] H.J. RACK, International Encyclopedia of Composites, Stuart M. Lee (Ed.), VCA Nueva York, 1990, vol. 4, p. 382.

[4] T. Das, P.R. Munroe y S. Bandyopadhyay, J. Mater. Sci. 31 (1996) 5351-5361.

[5] S.K. Varma, J. Ponce, M. Solís, S. Andrews y D.D. Salas, Metall. Mater. Trans. 27A (1996) 2023-2034.

[6] W.C. Chen, C.H.J. Davies, I.V. Samarasekera, J.K. Brimacombe y D.E.B. Hawolt, Metall. Mater. Trans. 27A (1996) 4095-4111.

[7] Borrego, J. IbÁÑez, V. López, M. Lieblich y G. GonZÁlez D., Scripta Mater. 34 (1996) 471-478.

[8] V.K. Varma, Y.R. Mahajan y V.V. Kutumbarao, Scripta Mater. 37 (1997) 485-489.

[9] S. Oin y M. Gupta, J. Mater. Sci. 30 (1995) 5223-5227.

[10] Ü. CöCEn, K. ÖNel y I. ÖZdemIR, Compos. Sci. Tech. 57 (1997) 801-807.

[11] A.W. Bowen, M. Ardakani y F.J. Humphreys, en Metal Matrix Composites: Processing, Microstructure and Properties, N. Hansen et al. (Eds.), Ris( National Laboratory, Denmark 1991, pp. 241-246.

[12] Chai-Yuan Sheu y Su-Jien Lin, Scripta Mater. 35 (1996) 1271-1276.

[13] N.L. Han, Z.G. Wang y G.D. Zhang, Compos. Sci. Tech. 57 (1997) 1491-1497.

[14] H. Li, J.B. Li, L.Z. Sun and Z.G. WANG, Compos. Sci. Tech. 57 (1997) 165-172.

[15] C.A. León y Robin A.L. Drew, Memorias del V Congreso Internacional de Materiales, Instituto Tecnológico de Saltillo, México, 1998, pp. 488-498.

[16] J.L. OrTiz, Tesis Doctoral, Escuela Técnica Superior de Ingenieros Industriales, Universidad Politécnica de Valencia, 2000.

[17] K. BUDINSKI, Engineering Materials, Properties and Selection, Prentice Hall, USA, 1996.

[18] M.F. Ashby y N.A. Waterman, The Materials Selector, Great Britain, Chapman \& Hall, GB, 1997.

[19] Shy-Wei Lai y D.D.L. Chung, J. Mater. Sci. 29 (1994) 6181-6198.

[20] B.S.S. Daniel y V.S.R. MurThy, Mater. Design, 16 (1995) 155-161.

[21] V. Jayaram, Mater. Eng. 3 (1992) 247-259.

[22] G.A. Edwards, K. Stiller, G.L. Dunlop y M.J. Couper, Acta Mater. 46 (1998) 3893-3904.

[23] V. Amigó, J.L. Ortiz y M.D. Salvador, Scripta Mater. 42 (2000) 383-388. 